\title{
Approximate Conformal Mappings and Elasticity Theory
}

\author{
Pyotr N. Ivanshin and Elena A. Shirokova \\ Kazan Federal University, 18 Kremlyovskaya Str., Kazan 420008, Russia \\ Correspondence should be addressed to Pyotr N. Ivanshin; pivanshi@yandex.ru
}

Received 4 June 2016; Revised 25 July 2016; Accepted 3 August 2016

Academic Editor: Vladislav Kravchenko

Copyright (C) 2016 P. N. Ivanshin and E. A. Shirokova. This is an open access article distributed under the Creative Commons Attribution License, which permits unrestricted use, distribution, and reproduction in any medium, provided the original work is properly cited.

Here, we present the new method of approximate conformal mapping of the unit disk to a one-connected domain with smooth boundary without auxiliary constructions and iterations. The mapping function is a Taylor polynomial. The method is applicable to elasticity problems solution.

\section{Introduction}

Conformal mappings play an important part in solution of elasticity theory problems if we apply to them complex variable theory. These investigations were started by Muskhelishvili [1]. Muskhelishvili considered multiple solution methods of certain plane problems in the cited monograph. In particular, he gave the solution technique for basic plane elasticity theory problems and that for the rods torsion. In order to do this, the author made an extensive use of conformal mappings from the unit disk to a given domain. The easiest solution appears in the case of the polynomial mapping.

Computer progress stimulated appearance of many numerical conformal mapping construction methods. Many of these methods were connected with the integral equation solutions. If we want to map a given simply connected domain to the disk, then we solve a linear integral equation either analytically or numerically (see, e.g., [2-5]). Note that if we want to map the disk to the given simply connected domain, then this problem solution turns out to be significantly harder and is traditionally reduced to nonlinear Theodorsen equation solution. The effective Wegmann method of this equation solution is based on iteration processes $[6,7]$.

The approximate conformal mapping of the unit disk to the given domain construction method presented here has the following advantages: it does not use any auxiliary constructions (triangulation, circle packing) or additional conformal mappings (zipper algorithm), it does not use accessory solutions of boundary value problems (conjugate function method, Wegmann method), and it even does not use iterations as Wegmann method or Fornberg method where the preimages of the given-on-the-unit circle points move along the given curve [8]. We solve the integral Fredholm equation. This equation is well known and allows us to construct the conformal mapping of the given domain onto the unit disk [9]. But we use this equation in order to find the monotone function that determines the necessary reparametrization of the given boundary. Moreover, our method provides us with the smooth solution in the form of Taylor polynomial. So it is possible to find the derivatives of the solutions. Also the method is connected with the domain boundary curve reparametrization described in [10].

The auxiliary function involved in the function inverse to the reparametrizing one can be found by integral equation solution. This solution is reduced to solution of the infinite system whose truncated form is regulated by two different polynomial coefficients calculation methods. If the system size is reasonable, then both formulas lead to close values of the desired coefficients. We present the example of nonconvex domain and construct the approximate conformal mapping of the unit disk onto this domain with the help of the boundary curve reparametrization.

The example of the unit disk mapping onto the hypotrochoid interior leads to solution of plane elasticity problems. With the help of the constructed approximate mapping functions, we analytically find the boundary shear stresses and draw the corresponding graphs. 
We also give the example of one boundary value problem solution for the corresponding domain which is reduced to finite linear equation system solution according to results of Muskhelishvili [1]. The approximate spline-interpolation solution of $3 \mathrm{D}$ boundary value problems for bars can be reduced to solution of a number of plane boundary value problems. We consider the 3D element of the bar with the hypotrochoidal cut and its deformation under the given boundary displacements at two levels.

\section{Approximate Conformal Mapping Construction by Means of the Boundary Reparametrization}

The method was introduced and theoretically described in [10]. Here, we give only the scheme of the function construction.

Consider a finite simply connected domain $D$ bounded by the smooth curve $L=\{z=z(t), t \in[0,2 \pi]\}$ and $z(0)=z(2 \pi)$. We trace the domain $D$ counterclockwise along $L$ as the parameter $t$ increases. We only deal with the cases in which the boundary $L$ representation is as follows:

$$
z(t)=x(t)+i y(t)=\sum_{k=-m}^{n} c_{k} e^{i k t}
$$

Note also that any smooth boundary may be approximated by a Fourier polynomial of this type.

If Fourier polynomial representation (1) of the curve $L$ possesses no summands with the negative degrees of $e^{i t}$, then the function that maps the unit disk onto the domain $D$ is immediately polynomial:

$$
z(\zeta)=\sum_{k=0}^{n} c_{k} \zeta^{k}
$$

Assume now that representation (1) contains nonzero coefficients $c_{-l}, l \in \mathbf{N}$. Then, it is possible to construct an approximate conformal mapping under reparametrization of (1) [10] leading to the coefficients $c_{-l}, l \in \mathbf{N}$, elimination.

In order to find this reparametrization $t(\theta), \theta \in$ $[0,2 \pi]$, we first construct the inverse function $\theta(t)=$ $\left.\arg (\zeta(z))\right|_{z=z(t) \in L}$.

Let $\zeta(z)$ be the analytic function that gives the conformal mapping of $D$ onto the unit domain so that $\zeta(0)=0$. The necessary condition for the function $\log (\zeta(z) / z)$ to be analytic in $D$ is just as in [10]:

$$
\begin{aligned}
q(t)= & \frac{1}{\pi} \int_{0}^{2 \pi} q(\tau)(\arg [z(\tau)-z(t)])_{\tau}^{\prime} d \tau \\
& +\frac{1}{\pi} \int_{0}^{2 \pi} \log |z(\tau)|(\log |z(\tau)-z(t)|)_{\tau}^{\prime} d \tau,
\end{aligned}
$$

where $q(t)=\alpha_{0}+\sum_{p=1}^{\infty} \alpha_{n} \cos n t+\beta_{n} \sin n t=\theta(t)-\arg z(t)$.

We consider the factor $\left(e^{i \tau}-e^{i t}\right)$ in the expression of $z(\tau)-$ $z(t)$ in order to separate the improper VP integral in the last integral equation. Finally, the function $q(t)$ is the solution of the Fredholm integral equation of the second kind:

$$
\begin{aligned}
q(t)= & \frac{1}{2 \pi} \int_{0}^{2 \pi} q(\tau) d \tau+\frac{1}{\pi} \int_{0}^{2 \pi} q(\tau) K(\tau, t) d \tau \\
& +\frac{1}{2 \pi} \int_{0}^{2 \pi} \ln |z(\tau)| \cot \frac{\tau-t}{2} d \tau \\
& +\frac{1}{\pi} \int_{0}^{2 \pi} \ln |z(\tau)| L(\tau, t) d \tau
\end{aligned}
$$

where

$$
\begin{aligned}
& K(\tau, t)=\operatorname{Im}\left[\ln \frac{z(\tau)-z(t)}{e^{i \tau}-e^{i t}}\right]_{\tau}^{\prime} \\
& =\operatorname{Im}\left[\ln \left(\sum_{k=1}^{n} c_{k} e^{i k t} \sum_{l=0}^{k-1} e^{i l(\tau-t)}-\sum_{j=1}^{m} c_{-j} e^{-i j \tau} \sum_{l=0}^{j-1} e^{i l(\tau-t)}\right)\right]_{\tau}^{\prime} \\
& L(\tau, t)=\operatorname{Re}\left[\ln \frac{z(\tau)-z(t)}{e^{i \tau}-e^{i t}}\right]_{\tau}^{\prime} \\
& =\operatorname{Re}\left[\ln \left(\sum_{k=1}^{n} c_{k} e^{i k t} \sum_{l=0}^{k-1} e^{i l(\tau-t)}-\sum_{j=1}^{m} c_{-j} e^{-i j \tau} \sum_{l=0}^{j-1} e^{i l(\tau-t)}\right)\right]_{\tau}^{\prime}
\end{aligned}
$$

are continuous operators.

Note that

$$
\begin{aligned}
& \ln [z(\tau)-z(t)] \\
& =\ln (2 i)+\ln \left(\sin \frac{\tau-t}{2}\right)+i \frac{\tau-t}{2} \\
& \quad+\ln \left(\sum_{k=1}^{n} c_{k} e^{i k t} \sum_{l=0}^{k-1} e^{i l(\tau-t)}-\sum_{j=1}^{m} c_{-j} e^{-i j \tau} \sum_{l=0}^{j-1} e^{i l(\tau-t)}\right),
\end{aligned}
$$

$\ln |z(\tau)|$

$$
=\sum_{j} \ln \left|1-\overline{z_{j}} e^{i \tau}\right|+\sum_{k} \ln \left|1-\frac{e^{i \tau}}{\widehat{z}_{k}}\right|+\sum_{k} \ln \left|\widehat{z}_{k}\right| .
$$

Here $z_{j}$ are the equation $\sum_{p=0}^{n+m} c_{p-m} z^{p}=0$ roots inside the unit disk, and $\widehat{z}_{k}$ are the roots of the same equation outside this disk.

Equation (4) is uniquely resolvable if we set the value $\alpha_{0}=$ $(1 / 2 \pi) \int_{0}^{2 \pi} q(\tau) d \tau=0$, for example. Indeed, this equation is the necessary condition for the function $\log (\zeta(z) / z)$ with boundary value $\log \left(e^{i \theta(t)} / z(t)\right)$ to be analytic in $D$.

Lemma 1. Let the numbers $j, p>1$ and a constant $U>0$ exist so that $\left|\partial^{j+p} G(\tau, t) / \partial t^{j} \partial \tau^{p}\right| \leq U$ and the function $Y(t)$ possess the bounded second derivative: $\left|Y^{\prime \prime}(t)\right|<T$. Then, the approximate solution of the uniquely resolvable Fredholm integral equation of the second kind

$$
X(t)=\int_{0}^{2 \pi} G(\tau, t) X(\tau) d \tau+Y(t),
$$


where $Y(t)$ is $2 \pi$ periodic and $G(\tau, t)$ is $2 \pi$ periodic with respect to both variables, can be reduced to solution of a finite linear system with error estimated by $\mathrm{O}\left(1 / N^{2}\right)$. Here, $N$ is the finite linear system rank.

Proof. We search for the solution $X(t)$ in the form of Fourier series and present the kernel $G(\tau, t)$ in the form of double Fourier series. Note that the restrictions on the kernel of integral equation and on the function $Y(t)$ yield the following estimates of the kernel Fourier coefficients, $\left|c_{n, m}\right|<U /|n|^{j}|m|^{p}$, and of $Y(t)$ Fourier coefficient $\left|\widetilde{c}_{k}\right|<$ $T /|k|^{2}$. We denote by $x$ the vector of the function $X(t)$ Fourier coefficients and by $y$ the vector of the function $Y(t)$ Fourier coefficients. Now the integral equation reduces to the infinite linear system which can be presented as follows:

$$
\left(\begin{array}{cc}
I_{N}+P_{N} & Q \\
R & I_{\infty}+S
\end{array}\right)\left(\begin{array}{l}
x_{1} \\
x_{2}
\end{array}\right)=\left(\begin{array}{l}
y_{1} \\
y_{2}
\end{array}\right) .
$$

Here, $P_{N}$ is an $N \times N$ matrix, $Q$ is an $N \times \infty$ matrix, $R$ is an $\infty \times N$ matrix, $S$ is an $\infty \times \infty$ matrix, and $I_{N}$ and $I_{\infty}$ are the identity matrices of relative sizes. Each of the vectors $x_{1}$ and $y_{1}$ has $N$ coordinates; the vectors $x_{2}$ and $y_{2}$ have the infinite number of coordinates. The Fourier coefficients of the smooth functions tend to zero as their numbers tend to infinity, so the coefficients of the matrices $Q, R$, and $S$ together with the coordinates of $y_{2}$ decrease rapidly as $N \rightarrow \infty$. Therefore, the matrix norm of $S$ and the vector norm of $y_{2}$ tend to zero as $N \rightarrow \infty$.

Let us prove that there exists the number $T \in \mathbb{N}$ such that the matrix operator $I_{N}+P_{N}$ is invertible $\forall N>T$ since the limit for $P_{N}$ integral operator $P$ is compact and the operator $I+P$ is invertible due to the lemma assumption. Note that we do not distinguish a finite dimensional vector and the Fourier polynomial with the corresponding finite coefficient set in our proof. Recall first that, due to chapter VI, paragraph 1 of [11], $\left\|P-P_{N}\right\| \rightarrow 0$ as $N \rightarrow \infty$. Assume that $\forall T \in \mathbb{N}$ there exists $s_{l}>T$ such that the spectrum of $P_{s_{l}}$ contains -1 . Then, there exists an infinite sequence $\left(v_{s_{l}}\right)_{l \in \mathbb{N}} \subset L^{2}$ such that $\left\|v_{s_{l}}\right\|=1$ and $P_{s_{l}} v_{s_{l}}=-v_{s_{l}}$. Let us prove that then there should exist at least one limit point for the sequence $\left\{v_{s_{l}}\right\}_{l \in \mathbb{N}}$. Since the operator $P$ is compact, there exist both a subsequence $\left\{v_{s_{k_{j}}}\right\}_{j \in \mathbb{N}}$ and an element $w_{0} \in L^{2}$ so that $P v_{s_{k_{j}}} \rightarrow w_{0},(j \rightarrow$ $\infty)$. Then, $\left\|P_{s_{k_{j}}} v_{s_{k_{j}}}-w_{0}\right\|=\left\|P_{s_{k_{j}}} v_{s_{k_{j}}}-P v_{s_{k_{j}}}+P v_{s_{k_{j}}}-w_{0}\right\| \leq$ $\left\|P_{s_{k_{j}}} v_{s_{k_{j}}}-P v_{s_{k_{j}}}\right\|+\left\|P v_{s_{k_{j}}}-w_{0}\right\| \leq\left\|P_{s_{k_{j}}}-P\right\|+\left\|P v_{s_{k_{j}}}-w_{0}\right\| \rightarrow 0$, $(j \rightarrow \infty)$. Thus, $\left\|v_{s_{k_{j}}}+w_{0}\right\|=\left\|-P_{s_{k_{j}}} v_{s_{k_{j}}}+w_{0}\right\| \rightarrow 0,(j \rightarrow \infty)$. Hence, $v_{s_{k_{j}}} \rightarrow-w_{0},(j \rightarrow \infty)$. Note that since $\left\|v_{s_{k_{j}}}\right\|=1$, $\forall j \in \mathbb{N}$, the element $w_{0}$ is nondegenerate. Let us show now that the relation $P w_{0}=-w_{0}$ holds true. Indeed, we have $\left\|P w_{0}+w_{0}\right\|=\left\|P w_{0}+P v_{s_{k_{j}}}-P v_{s_{k_{j}}}+w_{0}\right\| \leq\|P\|\left\|w_{0}+v_{s_{k_{j}}}\right\|$ $+\left\|-P v_{s_{k_{j}}}+P_{s_{k_{j}}} v_{s_{k_{j}}}-P_{s_{k_{j}}} v_{s_{k_{j}}}+w_{0}\right\| \leq\|P\|\left\|w_{0}+v_{s_{k_{j}}}\right\|$ $+\left\|P_{s_{k_{j}}} v_{s_{k_{j}}}-P v_{s_{k_{j}}}\right\|+\left\|w_{0}-P_{s_{k_{j}}} v_{s_{k_{j}}}\right\| \leq\|P\|\left\|w_{0}+v_{s_{k_{j}}}\right\|+$ $\left\|P-P_{s_{k_{j}}}\right\|+\left\|w_{0}+v_{s_{k_{j}}}\right\| \rightarrow 0,(j \rightarrow \infty)$. Hence, the spectrum of $P$ contains -1 , a contradiction with one of the assumptions.
We now take the number $N$ so that $\|S\|<1$ and the matrix $I_{N}+P_{N}$ possesses the inverse one. Now we have the relation

$$
\begin{aligned}
& \left(I_{N}+P_{N}\right)\left[I_{N}-\left(I_{N}+P_{N}\right)^{-1} Q\left(I_{\infty}+S\right)^{-1} R\right] x_{1} \\
& \quad=y_{1}-Q\left(I_{\infty}+S\right)^{-1} y_{2} .
\end{aligned}
$$

Obviously, one can choose the value of $N$ so large that $\left\|Q\left(I_{\infty}+S\right)^{-1} R\right\|_{2,2}=O\left(1 / N^{2}\right) \leq r$, where $r<1$ is arbitrary small. Now we estimate the norm of the difference between the solution $x_{1}$ and the solution $\tilde{x}_{1}$ of the truncated system $\left(I_{N}+P_{N}\right) \tilde{x}_{1}=y_{1}$ :

$$
\begin{aligned}
\left\|x_{1}-\tilde{x}_{1}\right\| \leq & \frac{1}{1-r}\left\|\left(I_{N}+P_{N}\right)^{-1}\right\|\left\|Q\left(I_{\infty}+S\right)^{-1}\right\|\left\|y_{2}\right\| \\
& +\frac{r}{1-r}\left\|\left(I_{N}+P_{N}\right)^{-1}\right\|\left\|y_{1}\right\| .
\end{aligned}
$$

Consider the first summand on the right-hand side of the last inequality. This is the summand that is determined by the operator approximation. The vector norm of $y_{2}$ can be estimated by $\mathrm{D}$. Jackson's inequality by $K / N^{2}$. The second summand (due to the series tail) also behaves no better than $O\left(1 / N^{2}\right)$. So the error is $O\left(1 / N^{2}\right)$.

We search for the integral equation solution in the form of the Fourier series:

$$
q(t)=\sum_{n=1}^{\infty} \alpha_{n} \cos (n t)+\beta_{n} \sin (n t) .
$$

The approximate solution then is the finite sum

$$
q(t)=\sum_{n=1}^{M} \alpha_{n} \cos (n t)+\beta_{n} \sin (n t) ;
$$

here, the number $M$ can be found from conditions that will be described later.

Now the approximate solution of the integral equation reduces to solution of the linear equation system

$$
\left(\begin{array}{cc}
A A & A B \\
B A & B B
\end{array}\right)\left(\begin{array}{l}
\alpha \\
\beta
\end{array}\right)=\left(\begin{array}{l}
F \\
G
\end{array}\right)
$$

here

$$
\begin{aligned}
& \alpha=\left(\begin{array}{c}
\alpha_{1} \\
\vdots \\
\alpha_{M}
\end{array}\right), \\
& \beta=\left(\begin{array}{c}
\beta_{1} \\
\vdots \\
\beta_{M}
\end{array}\right) .
\end{aligned}
$$


The vectors

$$
\begin{aligned}
& F=\left(\begin{array}{c}
f_{1} \\
\vdots \\
f_{M}
\end{array}\right), \\
& G=\left(\begin{array}{c}
g_{1} \\
\vdots \\
g_{M}
\end{array}\right)
\end{aligned}
$$

on the right-hand side of the system consist of elements

$$
\begin{aligned}
f_{l}= & \left(-\sum_{j} \operatorname{Im} z_{j}^{l}+\sum_{k} \operatorname{Im} \widehat{z}_{k}^{-l}\right) \frac{1}{l} \\
& +\frac{1}{\pi^{2}} \int_{0}^{2 \pi} \ln |z(\tau)| d \tau \int_{0}^{2 \pi} L(\tau, t) \cos (l t) d t, \\
g_{l}= & \left(\sum_{j} \operatorname{Re} z_{j}^{l}+\sum_{k} \operatorname{Re} \widehat{z}_{k}^{-l}\right) \frac{1}{l} \\
& +\frac{1}{\pi^{2}} \int_{0}^{2 \pi} \ln |z(\tau)| d \tau \int_{0}^{2 \pi} L(\tau, t) \sin (l t) d t, \\
& l=1, \ldots, M .
\end{aligned}
$$

The block matrices of size $M$

$$
A A, A B, B A, B B
$$

consist of elements

$$
\begin{aligned}
A A & =\left(\delta_{l n}-\frac{1}{\pi^{2}} \int_{0}^{2 \pi} \cos (n \tau) d \tau\right. \\
\cdot & \left.\int_{0}^{2 \pi} K(\tau, t) \cos (l t) d t\right)_{l, n=1}^{M} \\
A B & =\left(-\frac{1}{\pi^{2}} \int_{0}^{2 \pi} \sin (n \tau) d \tau\right. \\
\cdot & \left.\int_{0}^{2 \pi} K(\tau, t) \cos (l t) d t\right)_{l, n=1}^{M} \\
B A & =\left(-\frac{1}{\pi^{2}} \int_{0}^{2 \pi} \cos (n \tau) d \tau\right. \\
\cdot & \left.\int_{0}^{2 \pi} K(\tau, t) \sin (l t) d t\right)_{l, n=1}^{M} \\
B B & =\left(\delta_{l n}-\frac{1}{\pi^{2}} \int_{0}^{2 \pi} \sin (n \tau) d \tau\right. \\
\cdot & \left.\int_{0}^{2 \pi} K(\tau, t) \sin (l t) d t\right)_{l, n=1}^{M}
\end{aligned}
$$

here, $\delta_{l n}$ is the Kronecker delta function.
The numbers $m$ and $n$ are fixed. So the standard JenkinsTraub algorithm provides us with the given polynomial roots in $(1 / 2 \pi) \int_{0}^{2 \pi} \ln |z(\tau)| \cot ((\tau-t) / 2) d \tau$ calculation. Moreover, we can find this integral if we consider the Fourier decomposition of the function $\ln |z(t)|$. Really, if

$$
\ln |z(t)|=\gamma_{0}+\sum_{k=1}^{\infty} \gamma_{k} \cos k t+\delta_{k} \sin k t
$$

then according to the Hilbert formula

$$
\begin{aligned}
& \frac{1}{2 \pi} \int_{0}^{2 \pi} \ln |z(\tau)| \cot \frac{\tau-t}{2} d \tau \\
& \quad=\sum_{k=1}^{\infty}-\gamma_{k} \sin k t+\delta_{k} \cos k t
\end{aligned}
$$

So there is a way to omit the polynomial roots calculation.

The constructed function $\theta(t)$ must be monotone increasing. We construct the function inverse to it-the necessary reparametrization $t(\theta)$-with the help of FORTRAN spline function apparatus. According to Chapter 3 of [12], the algorithm computational complexity is $O\left(M^{3}\right)$. Assume now that we use $s$-digit base $\sigma$ floating point arithmetic. Then, formula (3.5.2) of [12] gives us the calculation estimate:

$$
\left\|\left(\begin{array}{l}
\widehat{\alpha} \\
\widehat{\beta}
\end{array}\right)-\left(\begin{array}{l}
\alpha \\
\beta
\end{array}\right)\right\|_{\infty} \leq \frac{1}{2} \sigma^{-s} \kappa_{\infty}\left(\begin{array}{cc}
A A & A B \\
B A & B B
\end{array}\right)\left\|\left(\begin{array}{l}
\alpha \\
\beta
\end{array}\right)\right\|_{\infty} .
$$

Here, $\left(\begin{array}{c}\widehat{\alpha} \\ \hat{\beta}\end{array}\right)$ is the computed solution.

This reparametrization $t(\theta)$ allows us to construct the function $z(\zeta),|\zeta|<1$, realizing the approximate conformal mapping on the given domain as a partial sum of the Taylor series:

$$
z(\zeta)=\sum_{j=1}^{\infty}\left(A_{j}+i B_{j}\right) \zeta^{j}
$$

here,

$$
\begin{aligned}
A_{j} & =\frac{1}{\pi} \int_{0}^{2 \pi} x(t(\theta)) \cos (j \theta) d \theta \\
& =\frac{1}{\pi} \int_{0}^{2 \pi} y(t(\theta)) \sin (j \theta) d \theta, \\
B_{j} & =-\frac{1}{\pi} \int_{0}^{2 \pi} x(t(\theta)) \sin (j \theta) d \theta \\
& =\frac{1}{\pi} \int_{0}^{2 \pi} y(t(\theta)) \cos (j \theta) d \theta, \quad j \in \mathbf{N} .
\end{aligned}
$$

Existence of two different coefficient $A_{j}$ and $B_{j}$ forms for the mapping function $z(\zeta)$ allows us to control the precision of the function $q(t)$ calculation by choosing the size $M$ of the block matrices and the number of Fourier polynomial coefficients for this function $q(t)$. 
We can also find the function $q(t), t \in[0,2 \pi]$, by solving the integral equation with respect to its derivative:

$$
\begin{gathered}
q^{\prime}(t)=-\frac{1}{\pi} \int_{0}^{2 \pi} q^{\prime}(\tau) \widetilde{K}(\tau, t) d \tau \\
+\left(\frac{1}{2 \pi} \int_{0}^{2 \pi} \ln |z(\tau)| \cot \frac{\tau-t}{2} d \tau\right. \\
\left.+\frac{1}{\pi} \int_{0}^{2 \pi} \ln |z(\tau)| L(\tau, t) d \tau\right)^{\prime},
\end{gathered}
$$

where

$$
\begin{aligned}
& \widetilde{K}(\tau, t) \\
& =\operatorname{Im}\left[\ln \left(\sum_{k=1}^{n} c_{k} e^{i k t} \sum_{l=0}^{k-1} e^{i l(\tau-t)}-\sum_{j=1}^{m} c_{-j} e^{-i j \tau} \sum_{l=0}^{j-1} e^{i l(\tau-t)}\right)\right]_{t}^{\prime} .
\end{aligned}
$$

Now the coefficients of the desired polynomial $z(\zeta)$ must be calculated via the formulas

$$
\begin{aligned}
A_{j} & =\frac{1}{\pi} \int_{0}^{2 \pi} x(t) \cos (j \theta(t)) \theta^{\prime}(t) d t \\
& =\frac{1}{\pi} \int_{0}^{2 \pi} y(t) \sin (j \theta(t)) \theta^{\prime}(t) d t, \\
B_{j} & =-\frac{1}{\pi} \int_{0}^{2 \pi} x(t) \sin (j \theta(t)) \theta^{\prime}(t) d t \\
& =\frac{1}{\pi} \int_{0}^{2 \pi} y(t) \cos (j \theta(t)) \theta^{\prime}(t) d t, \quad j \in \mathbf{N} .
\end{aligned}
$$

This way of the mapping function $z(\zeta)$ construction does not demand the inverse function $t(\theta)$. So it is better than the initial one when we use differentiation technics.

2.1. Examples of a Conformal Mapping to Nonconvex Domains. (1) We apply the conformal mapping construction method to the domain with the boundary parametrized as

$$
\begin{aligned}
z(t)= & 0.6+0.5 i+(0.95-0.4 i) e^{i t}+0.05 e^{-i t} \\
& +(0.4-0.2 i) e^{2 i t} .
\end{aligned}
$$

The corresponding domain is not convex and even not starlike with respect to the origin. The image of the unit disk $|\zeta|<1$ and the images of the curves $|\zeta|=$ const and $\arg \zeta=$ const are presented in Figure 1. Here, we construct the function $q$ as a polynomial of degree $M=200$. The constructed arrays $A$ and $B$ consist of $0.435195,0.217,0.0445382,-0.0644157,-0.122302,-0.136071$, $-0.108288,-0.0509652,0.0114895,0.0536582,0.0630275$, $0.0446863,0.0148454,-0.0102395,-0.0220771,-0.0210932$, $-0.0128945,-0.0035683,0.00312581,0.00613953,0.00613603$, $0.00435355, \quad 0.00194512,-0.000234746,-0.00165981$, $-0.00213913,-0.00179842,-0.000986218,-0.0001117$, and 0.000514721 and $-0.121471,-0.246766,-0.242473,-0.18307$, $-0.104088,-0.0205432,0.0508998,0.0906061,0.0887171$,

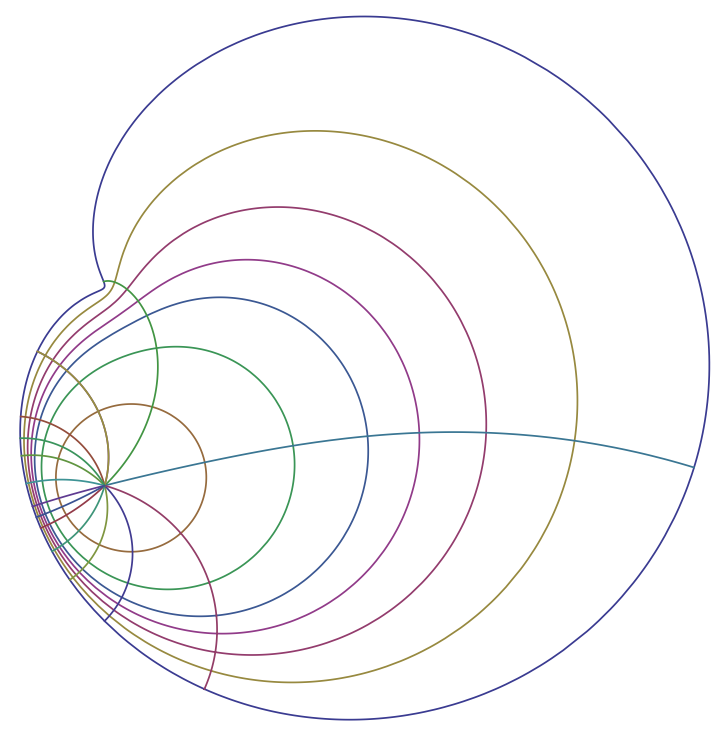

FIGURE 1: Nonconvex domain and the image of the regular polar net.

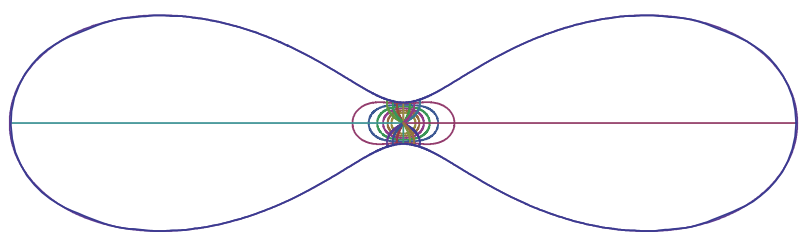

FIgURE 2: The curve and the image of the regular polar net.

$0.053735,0.0077085,-0.0271662,-0.0397708,-0.0324245$, $-0.0154014,0.000768445,0.0101997,0.0122604,0.00942934$, $0.00477677,0.000505807,-0.00232866,-0.00350766$, $-0.00328453,-0.00215714,-0.000716349, \quad 0.0005076$, $0.00118776,0.00126574$, and 0.000905516 , respectively.

The boundary point crowding in the example equals 75 to 1.

(2) Consider the approximation of the curve given by equation $z(t)=e^{i t}+0.6 e^{-i t}+0.3 e^{3 i t}$. The curve is similar to the curves considered in [8].

Again we construct the function $q$ as a polynomial of degree $M=200$. The array $A$ then consists only of odd elements $A_{2 j-1}, j=1,5000$. The array $B$ elements simply vanish. The boundary point crowding in the example in Figure 2 equals $1.3 * 10^{4}$ to 1 . The approximation to the initial curve is $O\left(10^{-3}\right)$. Note that in this case we need a resulting polynomial of degree $O(C)$; here, $C$ is the greatest possible boundary point crowding. Also this example shows us that there is no strong connection between $M$ and the border point crowding. The number $M$ determines the precision with which we construct either the monotone function $q$ or its derivative $q^{\prime}$ that we need for reparametrization.

The following section gives the example of the approximate conformal mapping applicable to the plane elasticity theory. 


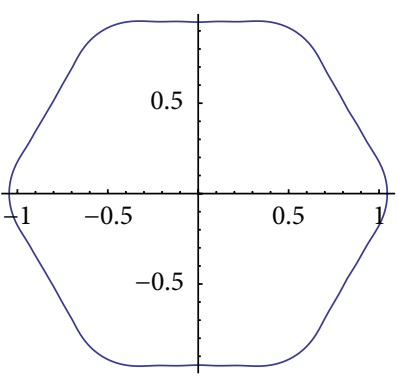

(a)

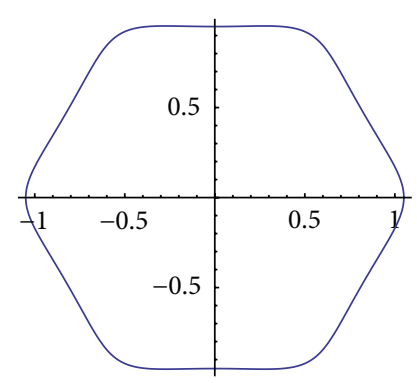

(b)

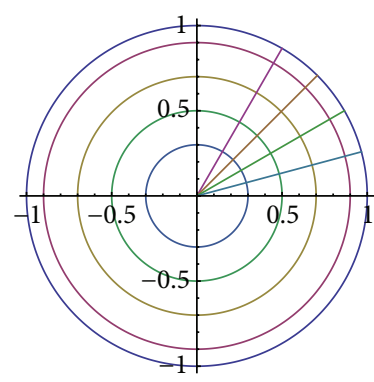

(c)

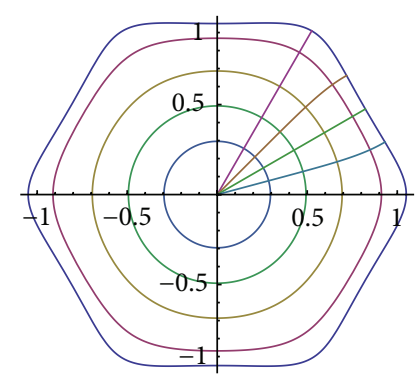

(d)

Figure 3: The mappings of the unit disk onto the hypotrochoidal interior: (a) hypotrochoid, (b) $P_{19}(\zeta)$ map of the unit disk, and (c) $P_{97}(\zeta)$ map of the unit disk.

\section{The Example of Conformal Mapping onto the Interior of the Hypotrochoid Close to the Hexagon with the Smoothened Angles}

Consider the complex parametric hypotrochoid equation:

$$
z(t)=e^{i t}+a e^{-5 i t}, \quad 0<a<0.2 .
$$

We fix $a=0.05$; item (a) of Figure 3 shows this hypotrochoid.

Now we construct the mapping of the unit disc onto this hypotrochoid interior applying the procedure described above. We solve (4) and find the polynomial mapping function. The mapping of the unit circle relatively well visually approximates the boundary curve of the hypotrochoid even for 19 first coefficients $\left(P_{19}(\zeta)\right)$ :

$$
\begin{aligned}
P_{19}(\zeta)= & 0.985399 \zeta+0.0441055 \zeta^{7}+0.0116172 \zeta^{13} \\
& +0.00429093 \zeta^{19} .
\end{aligned}
$$

The corresponding domain is given by item (b) of Figure 3.

Item (c) of Figure 3 demonstrates the map of the unit disk by the polynomial of the 97 th degree and the polar coordinate net transform under the mapping.

We also find the smooth solution after solving (24) and corresponding reparametrization of the given hypotrochoid. We name this polynomial function the smooth mapping.

3.1. Solution of the Torsion Problem for the Bar with the Hypotrochoidal Section. We examine the boundary shear stresses for the twisted bar with the hypotrochoidal interior as the cross section. We base the solution of the torsion problem on relation (13) of [1], Chapter 7: the value of the shear stress is proportional to the expression

$$
\frac{1}{\left|z^{\prime}\left(e^{i \theta}\right)\right|} \operatorname{Im}\left[e^{i \theta}\left[\phi^{\prime}\left(e^{i \theta}\right)-\overline{i z\left(e^{i \theta}\right)} z^{\prime}\left(e^{i \theta}\right)\right]\right],
$$

where $z(\zeta)$ is the polynomial mapping of the unit disk onto the hypotrochoid interior and $\phi(\zeta)$ is the analytic in the unit disk function with the boundary condition

$$
\operatorname{Im} \phi\left(e^{i \theta}\right)=\frac{\left|z\left(e^{i \theta}\right)\right|^{2}}{2} .
$$

The contour values of the tangent shear stress $T(t),-\pi<$ $t<\pi$, were found for the functions of the preceding section: for the mapping $P_{19}(\zeta)$, for the mapping $P_{97}(\zeta)$, and for the smooth mapping. Figure 4 shows the graphs of the function $T(t)$ for these mappings.

Each of the graphs shows that the minimal possible stress values happen in the points that correspond to the "hexagon vertices" and the maximal values are in the edges centers.

3.2. Solution of the Second Basic Elasticity Problem. We consider the second plane boundary value problem for a domain $D$ that is the image of the unit disk under the conformal mapping $z(\zeta)$. Due to results of [1], we can reduce the problem to finding the analytic in the unit disk functions $f(\zeta)$ and $g(\zeta),|\zeta|<1$, via the boundary condition ([1, Chapter 2, formula (1)])

$$
\left.\left(-\kappa f(\zeta)+\frac{z(\zeta)}{\overline{z^{\prime}(\zeta)}} \overline{f^{\prime}(\zeta)}+\overline{g(\zeta)}\right)\right|_{\zeta=e^{i \theta}}=P(\theta) ;
$$

here, $P(\theta), \theta \in[0,2 \pi]$, is the given function, $\kappa$ is the number depending on Lamé coefficients, $f(\zeta)=\phi(z(\zeta))$, and $g(\zeta)=$ $\psi(z(\zeta))$. The functions $\phi(z)$ and $\psi(z)$ are analytic in $D$ and are called the complex potentials of the plane elasticity theory [1]. The basic equation system for the polynomial mapping function $z(\zeta)$ was constructed in [1].

We solved this problem for the hypotrochoid interior in the case of $P(\theta)=0.01 e^{2 i \theta}, \kappa=2$. Figure 5 shows us the displacements of points of the concentric curves being the images of $|\zeta|=$ const.

3.3. Spline-Interpolation Solution of the 3D Second Basic Elasticity Problem. Consider now the 3D second basic elasticity theory problem for a bar $D \times[0, \bar{h}]$ parallel to $\mathrm{OH}$ axis in the space with coordinates $(x, y, h)$. We say that the spline-interpolation solution of this problem is the approximate solution which satisfies the elasticity equations and the boundary displacements at the finite number of levels $h=h_{j}, j=1, \ldots, n$, and approximates the displacements at the end faces of the bar. Assume that we construct a linear spline following [13]. Then, the solution is constructed for each element $D \times\left[h_{k}, h_{k+1}\right.$ in the linear form on $h$ components of the displacement vector-the functions 


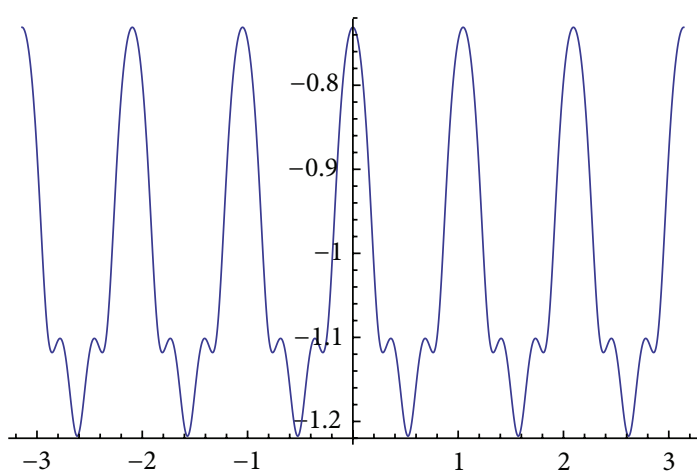

(a)

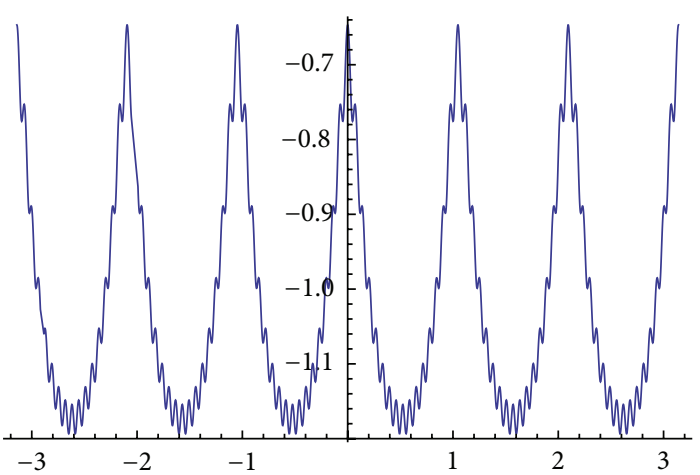

(b)

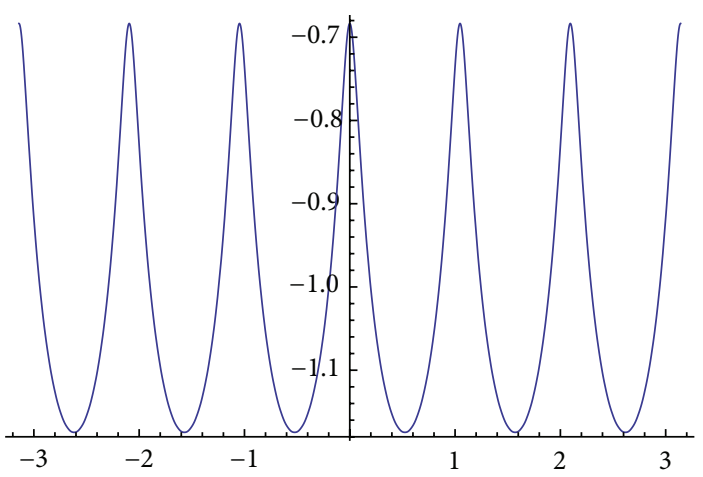

(c)

FIGURE 4: Shear stresses found for different functions: (a) for $P_{19}(\zeta)$, (b) for $P_{97}(\zeta)$, and (c) for smooth mapping.

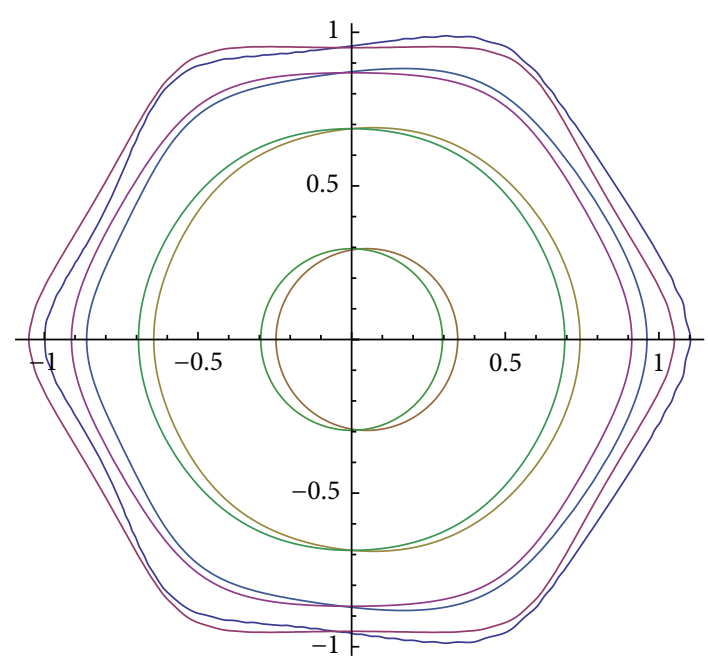

FIGURE 5: Hypotrochoid interior section and concentric inner circles displacements.

$u^{k}(x, y, h)+i v^{k}(x, y, h)=u_{0}^{k}(x, y)+i v_{0}^{k}(x, y)+h\left(u_{1}^{k}(x, y)+\right.$ $\left.i v_{1}^{k}(x, y)\right)$ and $w^{k}(x, y, h)=w_{0}^{k}(x, y)+h w_{1}^{k}(x, y)$. In order to find the functions $u_{j}^{k}(x, y)+i v_{j}^{k}(x, y), w_{j}^{k}(x, y), j=$ 0,1 , we apply the boundary conditions on the levels $h=$ $h_{k}$ and $h_{k+1}$. Then, the function $u_{1}^{k}(x, y)+i v_{1}^{k}(x, y)$ is a biharmonic function that meets equation coinciding with (32) and the function $w_{1}^{k}(x, y)$ is simply harmonic. The corresponding analytic functions can be restored via the boundary conditions. The functions $u_{0}^{k}(x, y)+i v_{0}^{k}(x, y)$ and $w_{0}^{k}(x, y)$ can also be restored via the respective conditions on their boundary values.

We construct as an example the element $D \times[0,1]$ where $D$ is the hypotrochoid interior. We assume that $u$ and $v$ meet the same boundary condition as in the case of the plane example on the level $h=1, u$ and $v$ vanish on the boundary of $D$ at the level $h=0$, and the third coordinate displacement $w$ also vanishes on the boundaries at both levels. Then, the function $u_{1}^{1}(x, y)+i v_{1}^{1}(x, y)$ is the same as that in the example of plane problem and $w_{1}^{1}(x, y) \equiv 0$. The other components of displacement vector can be calculated in terms of the variable $\zeta,|\zeta| \leq 1$. The displacements of the element upper (a) and lower (b) end faces are given by Figure 6.

Spline $h$ degree increase allows us not only to glue the adjacent element splines over the boundary of the common section but also to approximate these splines values over this common section in $L_{p}$ space.

The spline-interpolation solution is particularly effective in comparison with the FEM for the bodies with singular boundary points, for example, cones [14].

\section{Conclusion}

The conformal mapping method suggested in the paper is computationally efficient (of $\mathrm{O}\left(\mathrm{M}^{3}\right)$ computational complexity) and does not need any iterations. It provides us 

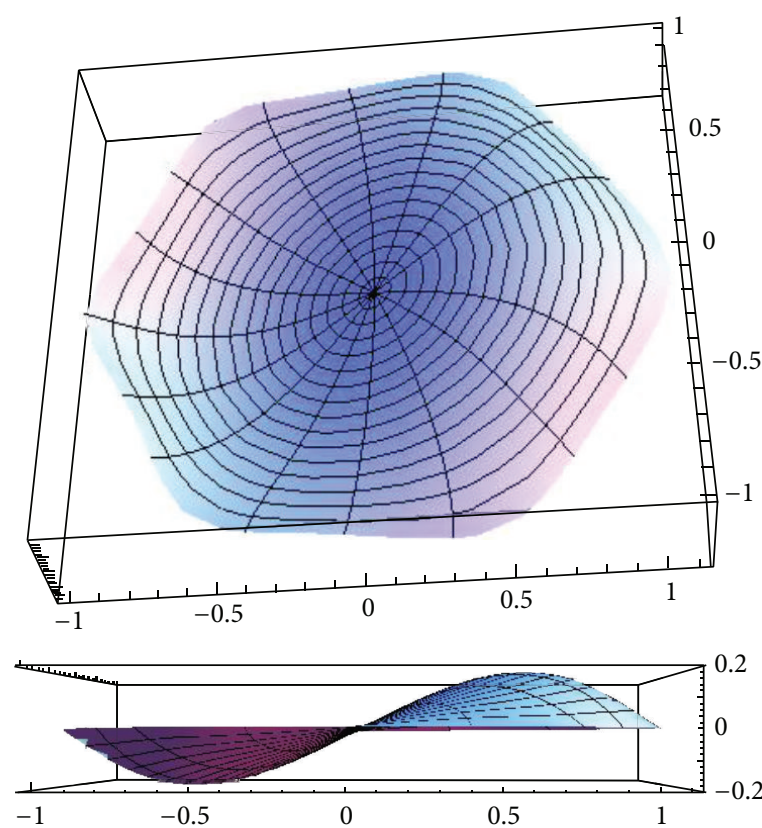

(a)

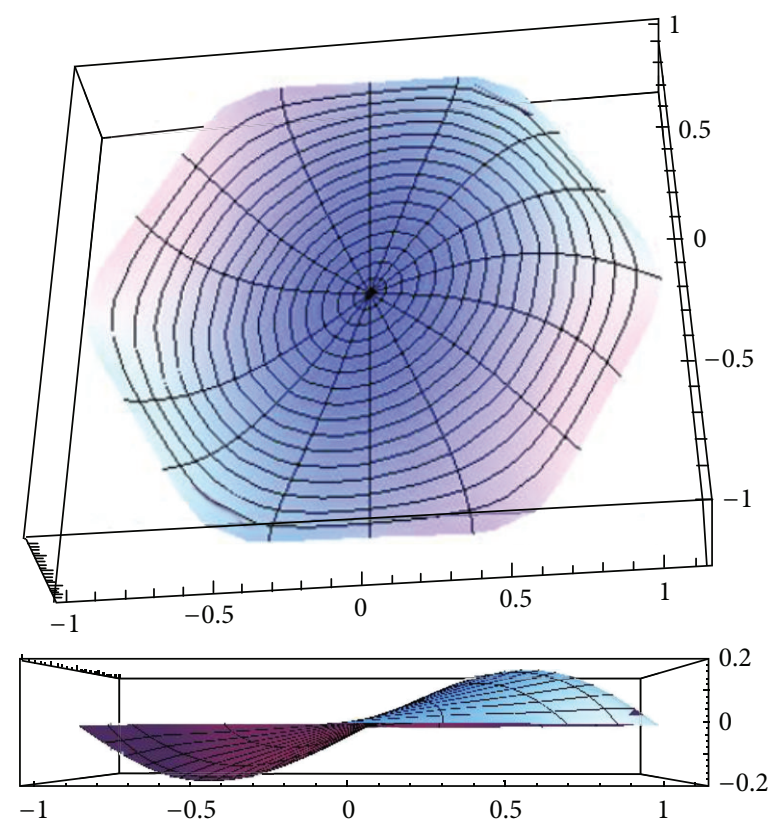

(b)

FIGURE 6: Top and bottom section deformations.

with the polynomial mapping function. This approximate conformal mapping method simplification for an arbitrary simply connected domain with smooth boundary makes it possible to apply the conformal mapping for many problems of elasticity. These problems reduce then to solution of linear equation systems.

\section{Competing Interests}

The authors declare that they have no competing interests.

\section{Acknowledgments}

The work was partly supported by the Russian Government Program of Competitive Growth of Kazan Federal University.

\section{References}

[1] N. I. Muskhelishvili, Some Basic Problems of the Mathematical Theory of Elasticity, Nauka, Moscow, Russia, 1966.

[2] B. C. Li and S. Syngellakis, "Numerical conformal mapping based on the generalised conjugation operator," Mathematics of Computation, vol. 67, no. 222, pp. 619-639, 1998.

[3] G. T. Symm, "An integral equation method in conformal mapping," Numerische Mathematik, vol. 9, pp. 250-258, 1966.

[4] T. K. DeLillo, "The accuracy of numerical conformal mapping methods: a survey of examples and results," SIAM Journal on Numerical Analysis, vol. 31, no. 3, pp. 788-812, 1994.

[5] D. M. Hough and N. Papamichael, "An integral equation method for the numerical conformal mapping of interior, exterior and doubly-connected domains," Numerische Mathematik, vol. 41, no. 3, pp. 287-307, 1983.
[6] R. Wegmann, A. H. M. Murid, and M. M. S. Nasser, "The Riemann-Hilbert problem and the generalized Neumann kernel," Journal of Computational and Applied Mathematics, vol. 182, no. 2, pp. 388-415, 2005.

[7] R. Wegmann, "An iterative method for conformal mapping," Journal of Computational and Applied Mathematics, vol. 14, no. 1-2, pp. 7-18, 1986.

[8] B. Fornberg, "A numerical method for conformal mappings," SIAM Journal on Scientific and Statistical Computing, vol. 1, no. 3, pp. 386-400, 1980.

[9] S. E. Warschawski, "On conformal mapping of variable regions," National Bureau of Standards Publications (NBS), vol. 18, pp. 175-187, 1952.

[10] E. A. Shirokova, "On approximate conformal mapping of the unit disk on an simply connected domain," Russia Mathematics (Iz VUZ), vol. 58, no. 3, pp. 47-56, 2014.

[11] L. A. Lyusternik and V. I. Sobolev, "The elements of functional analysis," Gosudarstv. Izdat. Tehn.-Teor. Lit., MoscowLeningrad, 1951.

[12] G. H. Golub and C. F. Van Loan, Matrix Computations, Johns Hopkins University Press, Baltimore, Md, USA, 1996.

[13] E. A. Shirokova, Spline-Interpolation Solution of One Elasticity Theory Problem, Bentham Science, Oak Park, Ill, USA, 2011.

[14] P. N. Ivanshin, L. R. Sekaeva, and E. A. Shirokova, "On the approximate solutions of the second basic elasticity theory problem," Lobachevskii Journal of Mathematics, vol. 31, no. 4, pp. 376-388, 2010. 


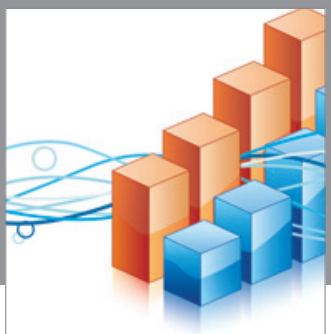

Advances in

Operations Research

vatem alat4

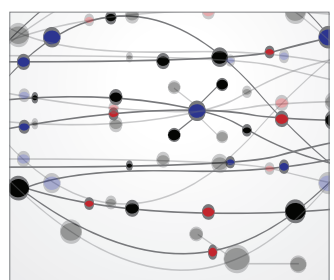

\section{The Scientific} World Journal
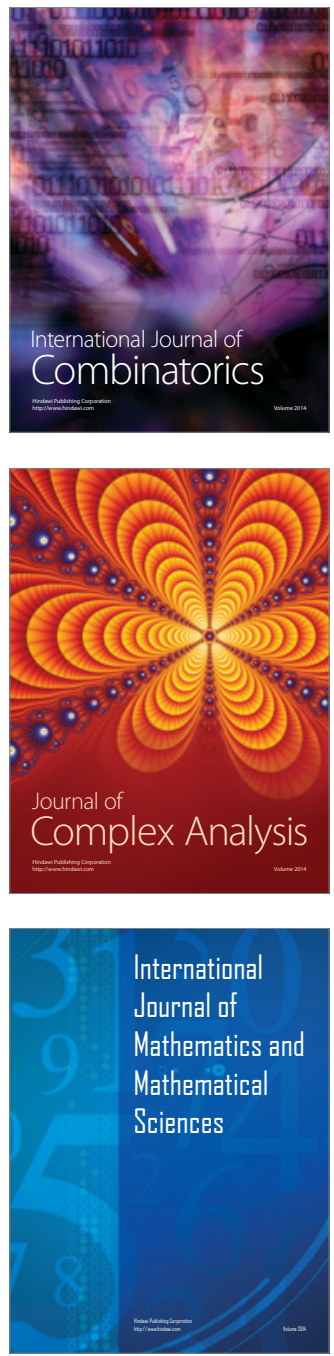
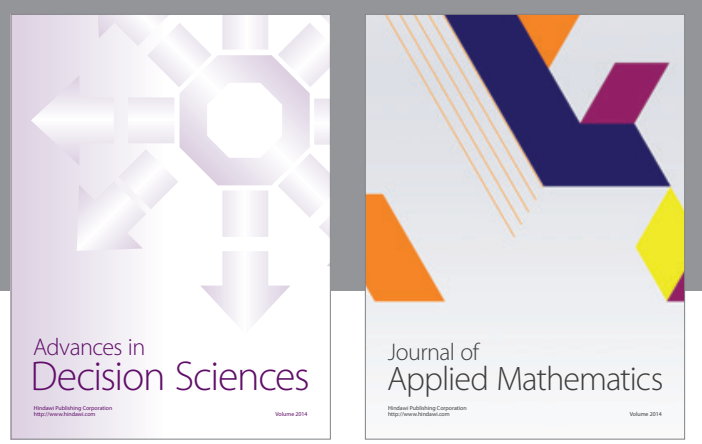

Algebra

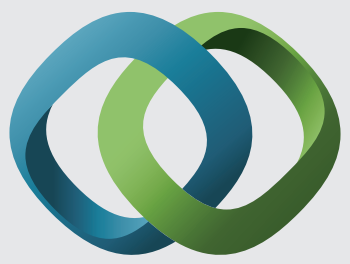

\section{Hindawi}

Submit your manuscripts at

http://www.hindawi.com
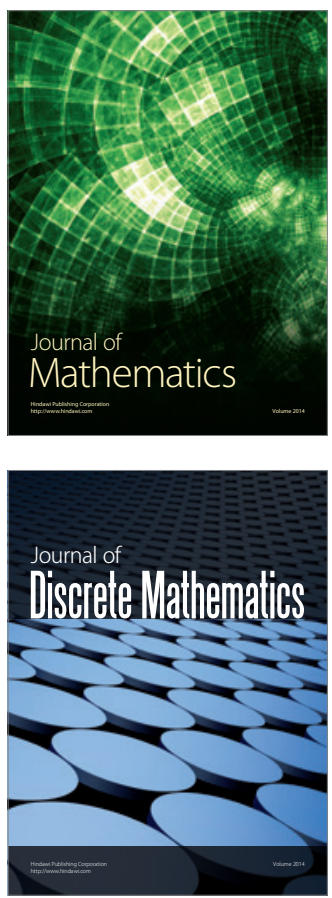

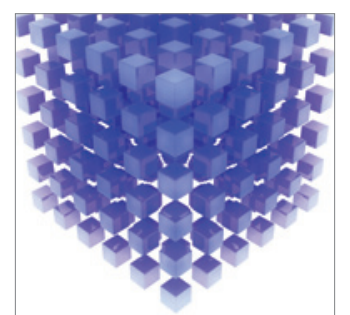

Mathematical Problems in Engineering
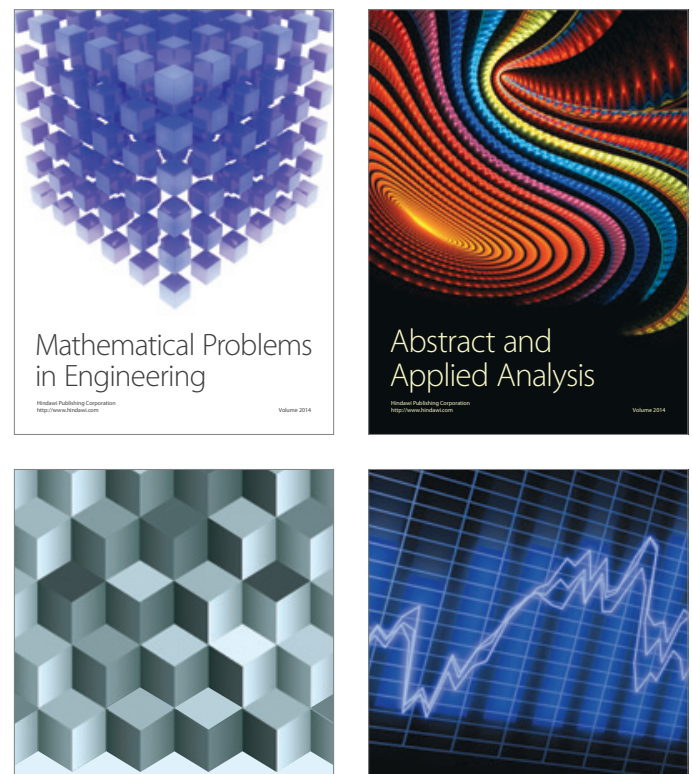

Journal of

Function Spaces

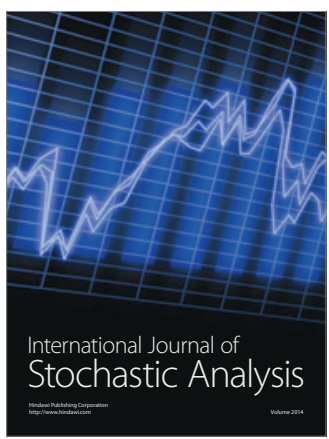

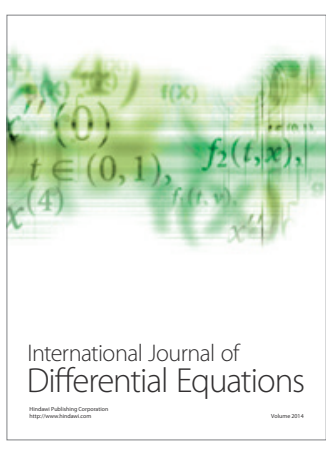
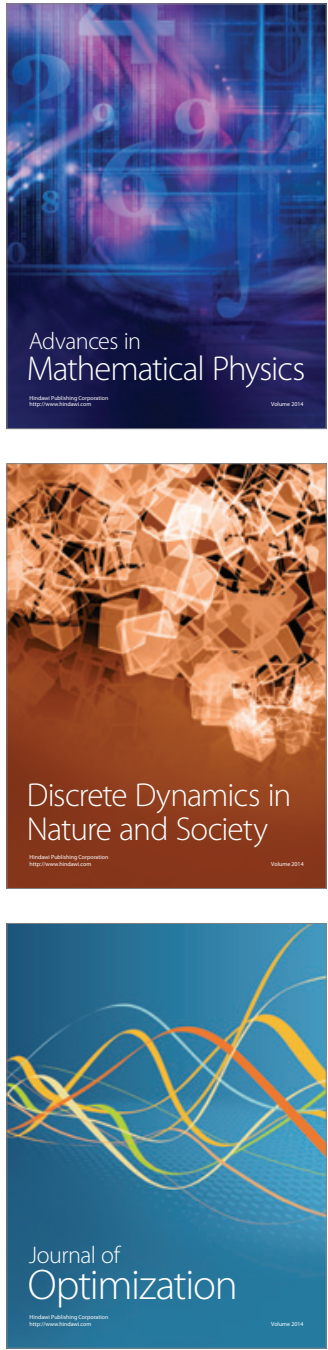